Research Article

\title{
An Economic Cost Assessment on HALEU Fuels for Small Modular Reactors
}

\author{
Liam Carlson, ${ }^{1}$ Zeyun Wu $\mathbb{D}^{1},{ }^{1}$ James Olson, $^{2}$ and Li (Emily) Liu ${ }^{2}$ \\ ${ }^{1}$ Department of Mechanical and Nuclear Engineering, Virginia Commonwealth University, 401 W. Main St., Richmond, \\ VA 23219, USA \\ ${ }^{2}$ Department of Mechanical, Aerospace, and Nuclear Engineering, Rensselaer Polytechnic Institute, $1108^{\text {th }}$ St., Troy, \\ NY 12180, USA \\ Correspondence should be addressed to Zeyun Wu; zwu@vcu.edu
}

Received 10 July 2020; Accepted 24 November 2020; Published 7 December 2020

Academic Editor: Zucchetti

Copyright ( 92020 Liam Carlson et al. This is an open access article distributed under the Creative Commons Attribution License, which permits unrestricted use, distribution, and reproduction in any medium, provided the original work is properly cited.

\begin{abstract}
Small modular reactors (SMRs) are currently being considered as future investments for commercial entities due to perceived advantages over traditional large-scale power reactors, particularly their considerably lower capital costs. One strategy for lowering the levelized cost of electricity (LCOE) of SMRs is to increase their burnup by utilizing high-assay low-enriched uranium (HALEU) fuels, which range from 5 to 20 weight percent (w/o) of U-235. By increasing fuel enrichment to HALEU levels, with higher specific fuel costs compared to standard enrichment, a plant may achieve an increased capacity factor by extending its fuel cycle and thereby reducing average yearly fuel supply costs. It is expected that the benefits of optimizing fuel enrichment to extend a reactor's fuel cycle outweigh the added cost due to more expensive fuel. In this paper, the net benefit of extending an SMR's fuel cycle by enriching uranium fuel to HALEU levels was estimated using 2017 nuclear fuel production market data with NuScale's 160 MWt SMR design as a case study. It was found that, for NuScale's design, plant LCOE decreased with increasing cycle length enabled by higher fuel enrichment. It was also observed that doubling cycle time from 24 months to 48 months netted each reactor a 1.23 \$/MWh reduction in LCOE. The total savings for a 12-module SMR design were estimated to be around $\$ 5,840,000$ per year. Therefore, utilizing HALEU fuel in SMRs can vastly improve their economic efficiency.
\end{abstract}

\section{Introduction}

Many small modular reactors (SMR) and microreactors are actively being pursued by various entities worldwide for a variety of applications, ranging from civilian applications to specialized military purposes $[1,2]$. The performance and economic market requirements for these reactors are not yet well defined, but one common trend observed among them is that nearly all the conceptual designs are considering the use of high-assay low-enriched uranium (HALEU) fuel in the potential near deployment. HALEU is generally defined for any uranium fuel that is enriched with U-235 w/o above $5 \%$ but lower than $20 \%$, which is above the NRC enrichment limit of 5\%. The potential commonality of HALEU utilization provides an opportunity for a broadly applicable economic cost study.
Since the application of HALEU fuels is primarily considered for potential economic benefits, it is necessary to understand how effective HALEU fuel may be in improving the economic efficiency of a nuclear power plant. The key economic benefits of using higher enriched fuel (compared to the current LWR fuels) include increasing revenue from a higher capacity factor of the reactor and reducing overall refueling costs. Higher enriched fuel has the potential to prolong a reactor's fuel cycle by increasing its maximum achievable fuel burnup, thus, improving its capacity factor with marginal effects on capital costs (one-time costs required for plant operation such as land, construction, and equipment) and operation and maintenance (O\&M) costs. By increasing the capacity factor, more energy can be supplied to the grid by a power plant for a longer duration. Longer durations of power generation reduce the refueling 
frequency which results in an improved reactor economy depending on the cost of fuel enrichment.

Although the overall price of fuel is expected to decrease due to higher burnup, the extra work needed to enrich fuel to HALEU levels causes the specific cost of fuel to increase. Since there is currently no existing HALEU supply chain, early batches may be cost-prohibitive for individual plants without long-term cost control factors. Additionally, the higher burnup inferred from increased enrichment suggests higher fuel fabrication costs as the fuel must be designed to mitigate increased rod internal pressure, cladding corrosion, rod, assembly growth, and cladding strain [3]. Moreover, enriching fuel to HALEU levels affects transportation costs; higher radiation and proliferation risks require increased standards for transportation and packaging regulations. From the reactor aspect, extending the cycle time with negligible changes in outage time from prolonged decay heat [4], the reactor pressure vessel (RPV) is exposed to more neutrons over a shorter cycle length. As a result, the RPV material will experience accelerated corrosion and embrittlement, causing a reduction in its operational lifetime. Because there are so many factors that affect the economics of implementing HALEU fuels in SMRs and microreactors, accurate cost estimations are difficult to achieve. Dynamic markets have a large effect on the economic viability of new reactor designs. For example, an increase in fuel costs may change the status of an SMR design from economically viable to cost prohibitive.

In order to assess the economic viability of increasing a reactor's fuel enrichment, a model for estimating plant levelized cost of electricity (LCOE) was developed. This model takes account of the changes in fuel enrichment and fuel cycle length. The NuScale's $160 \mathrm{MWt}$ SMR concept was chosen as a case study example to demonstrate the potential economic benefits of increasing fuel enrichment due to the following reasons. (1) It is far along in the NRC licensing process and can realistically start producing commercial power within 10 years. (2) It is based off standard PWR designs, of which, the economics have been thoroughly studied. Moreover, the results of this study can easily be related to other LWR designs. (3) It uses uranium oxide fuel, which has well-known fabrication costs, while the cost estimates of other novel fuel forms such as TRISO fuels contain high uncertainty. By estimating the economic benefits of increasing fuel enrichment on the NuScale's SMR design, the economic effect of HALEU fuels on SMRs can be better understood in general.

\section{LCOE Study Models}

The levelized cost of electricity (LCOE) for a nuclear power plant is the cost of electricity generated over a specified period of time, indicating the minimum revenue needed to achieve net benefit. The LCOE in electrical energy production can be considered as the present value of the price of the produced electrical energy, usually expressed in units of $\square / \mathrm{kWh}$ or $\$ / \mathrm{MWh}$, accounting for all lifetime costs of the plant system including the construction, O\&M, fuel costs, and other financial obligation of the project. Therefore, LCOE is a measure of the total cost of a power plant over the total energy produced for a cycle length and may be calculated using the equation below:

$$
\mathrm{LCOE}=\frac{C_{\mathrm{cc}}+C_{\mathrm{O \& M}}+C_{U}}{P \cdot \eta \cdot T_{\text {cycle }}},
$$

where $C_{\mathrm{cc}}, C_{\mathrm{O} \& \mathrm{M}}$, and $C_{U}$ represent the capital, O\&M, and fuel costs, respectively. It is important to note that a reactor cannot easily increase LCOE by simply extending its cycle time by reducing power production because the resulting increase in EFPD would be offset by lower revenue due to reduced power supplied to the grid. For this study, all reactor options are assumed to be operating at the same full-power except when refueling, where the power output is zero. The reactor cycle length and capacity factor are only influenced by varying potential fuel burnup as a result of changing fuel enrichment, namely, the change in LCOE is a result of varying fuel enrichments. As can be seen from equation (1), the LCOE can be readily broken into three terms where the last term is essentially the levelized cost of fuel (LCOF). Thus, it is possible to simplify the formula in equation (1) and estimate the LCOE without the knowledge of capital and O\&M costs. A simple and quick estimation of LCOE is then derived and expressed below:

$$
(\mathrm{LCOE})_{E}=\left[(\mathrm{LCOE})_{O}-(\mathrm{LCOF})_{O}\right] \frac{\eta_{O}}{\eta_{E}}+(\mathrm{LCOF})_{E}
$$

where $(\mathrm{LCOE})_{O}$ is the LCOE at standard enrichment, $(\mathrm{LCOF})_{O}$ is the LCOF at standard enrichment, and $\eta_{O}$ is the capacity factor at standard enrichment, while $(\mathrm{LCOE})_{E}$, $(\mathrm{LCOF})_{E}$, and $\eta_{E}$ represent the LCOE, LCOF, and capacity factor at updated enrichments. Equation (2) provides a ballpark LCOE estimation model and can be used to evaluate the overall effectiveness of fuel enrichments at various cycle lengths. It should be noted here that this model only accounts for capacity factor increase, fuel consumption rate reduction, and specific fuel cost increase and does not account for those case dependent factors such as transport cost increases.

To enable the calculations with equation (2), multiple parameters are required to estimate a reactor's LCOE. These parameters involve reported prices involved in ceramic fuel production and reactor design parameters. These values are displayed in Table 1. The fuel prices used for this study are approximate and were taken as of March 2017 [5]. The NuScale economic parameters were collected from publicly available sources except for the capacity factor and LCOF which were calculated [6-8].

Using publicly available design parameters for NuScale's 160 MWt SMR design [8] as summarized in the above 
TABLE 1: Fuel prices and parameters used for HALEU economic study.

\begin{tabular}{|c|c|c|c|}
\hline Fuel prices & $\begin{array}{c}\text { Process } \\
\text { Mining and milling } \\
\text { Conversion } \\
\text { Enrichment } \\
\text { Fuel fabrication }\end{array}$ & $\begin{array}{c}\text { Cost } \\
68 \\
105 \\
52 \\
300 \\
\end{array}$ & $\begin{array}{c}\text { Unit } \\
\$ / \mathrm{kg} \mathrm{U}_{3} \mathrm{O}_{8} \\
\$ / \mathrm{kgU} \\
\$ / \mathrm{SWU} \\
\$ / \mathrm{kg}\end{array}$ \\
\hline NuScale economic parameters & $\begin{array}{c}\text { Parameter } \\
\text { Thermal power, } P_{\text {th }} \\
\text { Electric power, } P_{e} \\
\text { Cycle burnup, } B \\
\text { Cycle length, } T_{\text {cycle }} \\
\text { Capacity factor, } \eta_{O} \\
\text { Cycle effective full-power days, } T_{\mathrm{EFPD}} \\
\text { Fuel loading mass, } M_{P} \\
\text { Average fresh load enrichment, } x_{P} \\
\text { Average levelized cost of electricity, LCOE } \\
\text { Estimated levelized cost of fuel, LCOF }\end{array}$ & $\begin{array}{c}\text { Value } \\
160 \\
45 \\
12 \\
24 \\
98.6 \\
720 \\
9213 \\
4.17 \\
86 \\
16.5-17.5\end{array}$ & $\begin{array}{l}\text { Unit } \\
\text { MWt } \\
\text { MWe } \\
\text { MWd/kgU } \\
\text { months } \\
\% \\
\text { days } \\
\text { kg } \\
\text { w/o } \\
\text { \$/MWh } \\
\text { \$/MWh }\end{array}$ \\
\hline
\end{tabular}

table, the LCOE can be estimated for product enrichments from 5-20 w/o and tails enrichments from $0.2-0.3 \mathrm{w} / \mathrm{o}$ for various fuel cycle lengths based on the LCOE developed in this section. It is worth mentioning that this economic model employed here is primarily based on the changes in fuel costs and capacity factors. The changes in capital, O\&M, decommissioning, and transport costs due to increased fuel enrichment were assumed to be negligible under this model.

The total fuel cost can be considered a summation of four costs: mining and milling, conversion, enrichment, and fuel fabrication. These four specific costs can each be multiplied by the material mass needed to produce one batch of fresh fuel $\left(\sim 1 / 3^{\text {rd }}\right.$ of the core) to find their respective total costs per cycle; the summation of these fuel manufacturing costs represents the total cost of fresh uranium in an equilibrium core loading, $C_{U}$. The economic effect on overall fuel costs from enriching fuel to HALEU levels is best interpreted by levelized cost of the fuel (LCOF) model, which can be used to calculate plant LCOE by assuming marginal changes in capital and O\&M costs. LCOF is defined as the total fuel costs divided by the energy produced in one cycle as illustrated in the below equation:

$$
\mathrm{LCOF}=\frac{C_{U}}{P \cdot T_{\mathrm{EFPD}}},
$$

where $P$ represents the electric power generated by the plant, and $T_{\text {EFPD }}$ represents the effective full-power days (EFPD), a plant is operated in one fuel cycle. The EFPD can be obtained by either subtracting the plant shutdown time from the plant cycle length or taking the product of a plant's cycle length and capacity factor. To better illustrate how the capacity factor affects LCOF, equation (4) may be further written as follows:

$$
\mathrm{LCOF}=\frac{C_{U}}{P \cdot \eta \cdot T_{\text {cycle }}},
$$

where $\eta$ is a power plant capacity factor, and $T_{\text {cycle }}$ is the total fuel cycle length in the unit of days. It can be seen that the LCOF will be affected by the fuel costs and the plant capacity factor.

The mining and milling costs consist of the extraction of natural uranium ore from the Earth and the process of converting it into pure $\mathrm{U} 3 \mathrm{O} 8$. This price is typically reported in dollars per kilogram of $\mathrm{U}_{3} \mathrm{O}_{8}$. The process of conversion involves chemical processes that convert solid $\mathrm{U}_{3} \mathrm{O}_{8}$ to a gaseous UF6 to prepare uranium for enrichment. The price of conversion is typically reported in dollars per kilogram of uranium. Due to the loss in the conversion process, $1.18 \mathrm{~kg}$ of $\mathrm{U}_{3} \mathrm{O}_{8}$ must be mined and milled for every kilogram of uranium converted. This natural uranium has an average enrichment of approximately $0.711 \mathrm{w} / \mathrm{o}$ and will act as the feedstock for the enrichment process, which is priced in units of dollars per separative work unit (SWU). Separative work is a measure of the amount of energy required to enrich the uranium and may be calculated using equation (5) as follows [9]:

$$
\begin{aligned}
\mathrm{SW} & =M_{W}\left(2 x_{w}-1\right) \ln \left(\frac{x_{w}}{1-x_{w}}\right) \\
& +M_{P}\left(2 x_{P}-1\right) \ln \left(\frac{x_{P}}{1-x_{P}}\right)-M_{F}\left(2 x_{F}-1\right) \ln \left(\frac{x_{F}}{1-x_{F}}\right)
\end{aligned}
$$

where $x_{P}, x_{W}$, and $x_{F}$ are the respective product, waste, and feed enrichments; $M_{P}, M_{W}$, and $M_{F}$ are the respective product, waste, and feed masses. To facilitate the evaluation of the price of a specific fuel product, the product mass 
can be assumed to be one kilogram. In this case, the feedto-product and waste-to-product ratios become the feed and product masses, so $M_{F}$ and $M_{W}$ are equal to $M_{F} / M_{P}$ and $M_{w} / M_{P}$, which can be considered as functions of enrichment as shown in equations (6) and (7), respectively [9]:

$$
\begin{aligned}
& \frac{M_{F}}{M_{P}}=\frac{x_{P}-x_{W}}{x_{F}-x_{W}}, \\
& \frac{M_{W}}{M_{P}}=\frac{x_{P}-x_{W}}{x_{F}-x_{W}} .
\end{aligned}
$$

By substituting equations (6) and (7) into (5), a formula for finding the separative work in SWUs can be derived as a function of enrichments. The cost of enriching one kilogram of product, $c_{U E}$, can thus be calculated by relating the separative work of enrichment to the price in dollars per SWU (the coefficient $\mathrm{C}_{\mathrm{SWU}}$ is mentioned in the equation below):

$$
\begin{aligned}
c_{U E} & =\mathrm{C}_{\mathrm{SWU}}\left[\left(\frac{x_{P}-x_{W}}{x_{F}-x_{W}}\right)\left(2 x_{w}-1\right) \ln \left(\frac{x_{w}}{1-x_{w}}\right)\right. \\
& \left.+\left(2 x_{P}-1\right) \ln \left(\frac{x_{P}}{1-x_{P}}\right)-\left(\frac{x_{P}-x_{W}}{x_{F}-x_{W}}\right)\left(2 x_{F}-1\right) \ln \left(\frac{x_{F}}{1-x_{F}}\right)\right]
\end{aligned}
$$

As can be seen from equation (8), it is not necessary to know the feed or waste masses to find the necessary separative work to enrich uranium fuel to a certain level. As a result, the economics of enrichment have a significant dependence on tails enrichment, or the enrichment of depleted uranium to be discarded or used for other purposes. To estimate fuel price, it is necessary to know the feed factor to determine how much material must be mined and milled, converted, and enriched to produce one kilogram of uranium. For this model, it is assumed that a reliable HALEU supply chain has been established; this would suggest that the NuScale reactor modified for HALEU fuel is an $n$-th of a kind reactor. Additionally, the total mining, milling, and conversion costs must be considered into the enrichment feed factor to determine the mass of $\mathrm{U}_{3} \mathrm{O}_{8}$ and natural uranium needed to produce one kilogram of the product.

With all the above costs being considered, only the other cost for fuel production is the fuel fabrication costs, also known as the cost of converting enriched uranium from its gaseous form, UF6, to its final fuel form. In the case of NuScale, like that of most PWRs, the fuel is in the form of ceramic UO2 fuel pellets. Fabrication prices range from $\$ 200$ to $\$ 400$ per kilogram of uranium and are higher for fuels designed to withstand higher burnup. The average fabrication cost is around $300 \$ / \mathrm{kgU}$ [5].

\section{LCOE Study Results}

The focus of this study is the impact of higher enrichment on fuel price. If an increase in total fuel cost overpowers the impact of extending cycle length, then a reactor cannot justify increasing its enrichment. By summing the fuel costs from mining and milling, conversion, enrichment, and fabrication for an equilibrium fuel loading, the total fuel cost for NuScale's 160 MWt SMR design was estimated to range from 12.8 to 13.6 million USD (U.S. dollars) as shown in Table 2. In addition, Table 2 shows the range of total fuel costs based on the possible product and tails enrichments for HALEU fuel production. The minimum total fuel cost for HALEU fuels, 15.2 million USD, corresponds to the lowest product and tails enrichments at $5 \mathrm{w} / \mathrm{o}$ and $0.2 \mathrm{w} / \mathrm{o}$ respectively. Likewise, the maximum total fuel cost, 62.7 million USD, corresponds with the highest product and tails enrichment at $20 \mathrm{w} / \mathrm{o}$ and $0.3 \mathrm{w} / \mathrm{o}$. Assuming the input price data, all have $1 \%$ uncertainty; the lowest and highest possible total core loading fuel costs contain uncertainties of $0.57 \%$ and $0.64 \%$, respectively. With an input error of $5 \%$, these values become $2.9 \%$ and $3.2 \%$, respectively. This cost increase is due to the increased separative work required for enrichment as well as the increased supply of uranium ore needed to produce one kilogram of fuel. Moreover, total fuel costs diverge with increasing product enrichment. While the fuel costs at $5 \mathrm{w} / \mathrm{o}$ have a range of 0.9 million USD, fuel costs at $20 \mathrm{w} / \mathrm{o}$ have a range of 4.4 million USD depending on tails enrichment. Higher product enrichments have increased sensitivity to changes in tails enrichment. The percent relative range is logarithmically correlated with product enrichment. At $5 \mathrm{w} / \mathrm{o}$ product enrichment, the percent relative range is $6.3 \%$; while at $20 \mathrm{w} / \mathrm{o}$, it is $7.3 \%$.

The average LCOE estimated for NuScale's SMR is approximately $86 \$ / \mathrm{MWh}$ [10]. The increase in total fuel costs due to higher enrichment may only be considered economically viable if the resulting LCOE is not more than the original design and competing power generation technology options available to power generation equipment owners. In other words, favorable options are those where the energy produced in a power plant increases at a higher factor than that of the costs. Although more energy can always be supplied with an increased fuel cycle length due to higher enrichments, the increase in the cycle length is only economically viable below a certain enrichment. Past that point, the LCOE becomes too high due to specific fuel costs of high fuel enrichments. Figure 1 illustrates this concept by displaying the estimated LCOE for HALEU level enrichments of cycle lengths of 2.5,3,3.5, and 4 years assuming a fixed tails enrichment of $0.2 \mathrm{w} / \mathrm{o}$. This tails enrichment was chosen because it represents the lowest possible cost option given the range of typical enrichments as shown in Table 2. Equation (2), which is used to calculate the curves shown in Figure 1, provides economic assessment and does not 
TABLE 2: Total core load uranium cost (with units of million USD\}.

\begin{tabular}{|c|c|c|c|c|c|c|c|c|c|c|c|}
\hline & & \multicolumn{9}{|c|}{ Product Enrichment $\{$ w/o $\}$} & NuScale Enrichment \\
\hline \multirow{7}{*}{ Tails enrichment $\{\mathrm{w} / \mathrm{o}\}$} & & 5.0 & 7.0 & 9.0 & 11.0 & 13.0 & 15.0 & 17.0 & 19.0 & 20.0 & 4.17 \\
\hline & 0.20 & 15.2 & 20.8 & 26.5 & 32.3 & 38.0 & 43.8 & 49.6 & 55.4 & 58.3 & 12.8 \\
\hline & 0.22 & 15.3 & 21.0 & 26.7 & 32.5 & 38.3 & 44.2 & 50.0 & 55.8 & 58.8 & 12.9 \\
\hline & 0.24 & 15.4 & 21.2 & 27.0 & 32.9 & 38.8 & 44.7 & 50.6 & 56.5 & 59.4 & 13.0 \\
\hline & 0.26 & 15.6 & 21.5 & 27.4 & 33.4 & 39.3 & 45.3 & 51.3 & 57.3 & 60.3 & 13.2 \\
\hline & 0.28 & 15.8 & 21.8 & 27.9 & 33.9 & 40.0 & 46.1 & 52.2 & 58.3 & 61.4 & 13.4 \\
\hline & 0.30 & 16.1 & 22.3 & 28.4 & 34.6 & 40.8 & 47.1 & 53.3 & 59.6 & 62.7 & 13.6 \\
\hline
\end{tabular}

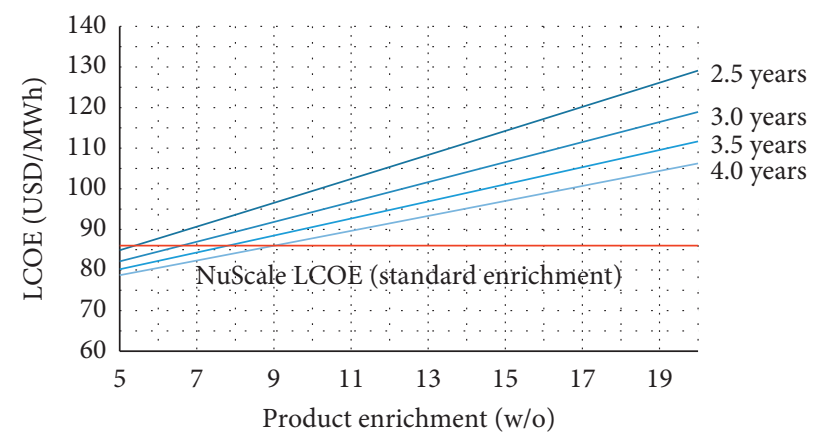

Figure 1: Projected LCOE of target enrichments and cycle lengths (the red line titled "NuScale LCOE (standard enrichment)" refers to the estimated LCOE for the NuScale's current SMR design).

account for potential practical restrictions of the NuScale's SMR design.

Figure 1 shows that a four-year cycle at $5 \mathrm{w} / \mathrm{o}$ enrichment can achieve the lowest LCOE; however, a four-year cycle is ultimately unachievable at that enrichment without reducing power or significantly altering core design. If the optimized enrichment required to extend the cycle life of NuScale's SMR falls to the right of the intersection between the red line and the target cycle length's LCOE curve, it would not be economically beneficial to extend the cycle length. The resulting LCOE would fall above 86 \$ $/ \mathrm{MWh}$. The upper limit of economically viable enrichments is $5.4,6.6$, 7.8 , and $9.0 \mathrm{w} / \mathrm{o}$ for cycle lengths of $2.5,3,3.5$, and 4 years respectively. These values suggest a direct linear relationship between economic upper limits of enrichment and cycle time. Extrapolating this trend implies the maximum HALEU enrichment of $20 \mathrm{w} / \mathrm{o}$ could operate economically for a maximum cycle of approximately 8.6 years.

The economic study performed above provides an economic standing for a final reactor design. As implied by the case study, with the given enrichment of the equilibrium core, an estimated annual saving compared to the original NuScale SMR design can be made. By employing the Studsvik Scandpower LWR reactor analysis code suites [11], a revised core loading for a $160 \mathrm{MWt}$ SMR can be optimized for a 48-month fuel cycle with HALEU fuels. Nothing is altered in the revised core loading other than loading fuel enrichment. The revised design employed the NuScale's equilibrium 3-batch out-in the loading scheme, in which the core's center assembly is replaced every cycle, while 12 remaining fresh assemblies are reshuffled to once-burned then twice-burned batches [6]. With only physics considerations and using an equilibrium fresh-batch loading of 6 assemblies at $9.10 \mathrm{w} /$ o, 6 assemblies at $8.1 \mathrm{w} / \mathrm{o}$, and 1 center-core assembly at $5.2 \mathrm{w} / \mathrm{o}$, the average equilibrium fresh fuel enrichment is found to be $8.34 \mathrm{w} / \mathrm{o}$.

Based on the LCOE data shown in Figure 1, the HALEU SMR design mentioned above is estimated to operate at an LCOE of $84.8 \$ / \mathrm{MWh}$, or $1.23 \$ / \mathrm{MWh}$ less than that of NuScale's 24-month design. By assuming a linear reactivity model (LRM) [12], a relationship for enrichment and cycle length was established by comparing NuScale data to the simulated SMR 48-month cycle results. Figure 2 illustrates this relationship. By interpolating the LRM, the optimal core loading enrichments for 30, 36, and 42-month cycles were found to be $5.21,6.26$, and $7.30 \mathrm{w} / \mathrm{o}$, while their corresponding LCOEs were 85.5, 85.2, and 84.9 \$/MWh, respectively. The red and yellow error bars represent errors corresponding to input price data uncertainties of $1 \%$ and $5 \%$, respectively. The trends observed in Figure 2 suggest that the improvement in LCOE from the original 24-month cycle due to increased enrichment becomes less significant with each step in cycle time. The approximated data points from 2.5 to 3.5 years were derived using an LRM; because of this, the corresponding data are not as well defined as those derived from a more rigorous physics model (like the model used to simulate the 4-year case). 


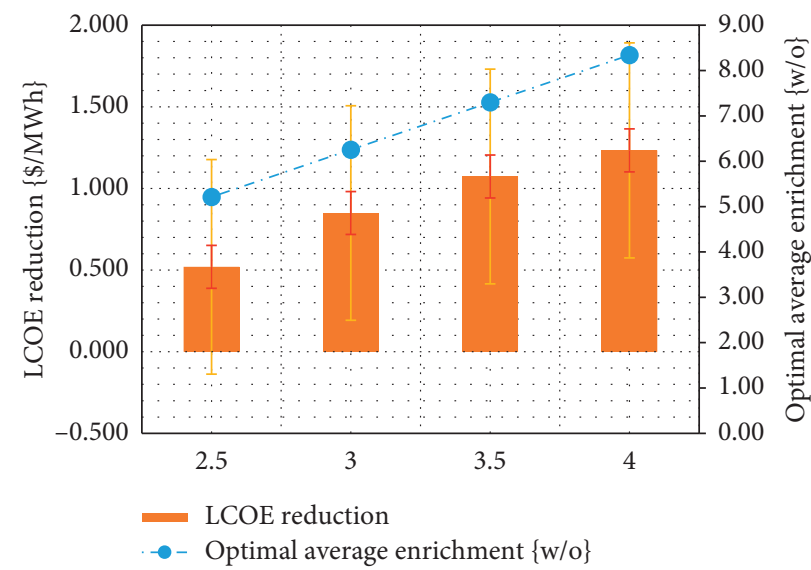

FIGURE 2: Optimized enrichments and LCOE reduction for SMR with extended cycle length.

\section{Conclusions}

With a power output of $45 \mathrm{MWe}$, the yearly savings for one 48-month cycle reactor using HALEU fuel is estimated to be $\$ 486,000$. With 12 modules proposed for NuScale's power plant, the total yearly savings are expected to be roughly $\$ 5,840,000$. However, this final value may fluctuate greatly with market changes in fuel cost. For every $1 \%$ increase in the fuel prices listed in Table 1 , there is expected to be a corresponding $10.7 \%$ increase in total plant savings. This is something to keep in mind when analyzing the long-term economic effects of implementing HALEU fuel into the design of a reactor. However, it is shown that increasing the fuel enrichment to extend cycle length to 48 months may greatly increase the economic efficiency of an SMR. While many implications such as lack of current HALEU manufacturing infrastructure and increased fuel transport costs may diminish the benefits of HALEU fuels, it is still a very promising step in furthering the economic advantages of nuclear power.

Since the economic study does not factor in the effects of increased transport costs and lack of current HALEU production infrastructure, the LCOE curves are likely underestimated and the maximum economically viable enrichments are likely overestimated. Despite this, the LCOE study provides estimated values for determining the economic effects of increasing fuel with various HALEU levels. Although increasing fuel enrichment can extend the cycle length, this relationship varies between reactor designs. To promise the economic benefits of extending cycle length for NuScale's SMR design, the corresponding optimal fuel enrichment is required and must be obtained with indepth reactor analysis. With more definite knowledge, the total savings due to increased enrichment may be estimated more precisely.

\section{Data Availability}

The data used to support the findings of this study are included in the article.

\section{Conflicts of Interest}

The authors declare that they have no conflicts of interest.

\section{References}

[1] E. McGinnis, The BIG Potential for Nuclear Microreactors, 2019, https://www.energy.gov.

[2] C. Charles, Micro-reactors could power remote military bases within a decade, 2018, https://www.energy.gov.

[3] F. Pimentel, The Economic Benefits and Challenges with Utilizing Increased Enrichment and Fuel Burnup for LightWater Reactors, NEI, Washington, DC, USA, 2019.

[4] R.B Pond and J.E Matos, Nuclear Mass Inventory, Photon Dose Rate and Thermal Decay Heat of Spent Research Reactor Fuel Assemblies, Argonne National Laboratory, Argonne, IL, USA, 1996.

[5] C. P. Pannier and R. Škoda, "Small modular reactor and large nuclear reactor fuel cost comparison," in Proceedings of the 22nd International Conference on Nuclear Engineering, Prague, Czech Republic, November 2014.

[6] NuScale, Cost-Competitive Energy, NuScale, Corvallis, OR, USA, 2019.

[7] U.S. NRC, NuScale Plant Design Overview, U.S. NRC, Rockville, Maryland, USA, 2012.

[8] U.S. NRC, Refueling Operations Report for NuScale Power Module, U.S. NRC, Rockville, MD, USA, 2009.

[9] World Nuclear Association, Economics of Nuclear Power, World Nuclear Association, London, UK, 2020.

[10] NuScale Power, Upgrading America's Energy System, NuScale, Corvallis, OR, USA, 2017.

[11] J. Rhodes, K. Smith, and D. Lee, CASMO-5 Development and Applications, Studsvik Scandpower Inc., Wilmington, NC, USA, 2006.

[12] G. T. Parks, "A piecewise linear reactivity fuel-cycle model," Annals of Nuclear Energy, vol. 16, no. 8, pp. 417-422, 1989. 